\title{
Experience Modifies Olfactory Acuity: Acetylcholine-Dependent Learning Decreases Behavioral Generalization between Similar Odorants
}

\author{
Max L. Fletcher and Donald A. Wilson \\ Department of Zoology, University of Oklahoma, Norman, Oklahoma 73019
}

Perceptual learning has been demonstrated in several thalamocortical sensory systems wherein experience enhances sensory acuity for trained stimuli. This perceptual learning is believed to be dependent on changes in sensory cortical receptive fields. Sensory experience and learning also modifies receptive fields and neural response patterns in the mammalian olfactory system; however, to date there has been little reported evidence of learned changes in behavioral olfactory acuity. The present report used a bradycardial orienting response and crosshabituation paradigm that allowed assessment of behavioral discrimination of nearly novel odorants, and then used the same paradigm to examine odorant discrimination after associative olfactory conditioning with similar or dissimilar odorants.
The results demonstrate that associative conditioning can enhance olfactory acuity for odors that are the same as or similar to the learned odorant, but not for odors dissimilar to the learned odorant. Furthermore, scopolamine injected before associative conditioning can block the acquisition of this learned enhancement in olfactory acuity. These results could have important implications for mechanisms of olfactory perception and memory, as well as for correlating behavioral olfactory acuity with observed spatial representations of odorant features in the olfactory system.

Key words: adaptation; perceptual learning; piriform cortex; olfaction; olfactory memory; scopolamine
Experience can shape both behavioral and physiological responses to sensory input. Both associative conditioning and, in some cases, simple stimulus exposure have been shown to modify receptive fields of sensory neurons and associated behavioral sensory abilities in most sensory modalities (Gibson, 1953; Gilbert et al., 2001). For example, training an owl monkey to discriminate between different frequencies of punctate tactile stimulation of the finger both modifies somatosensory cortex single-unit response patterns to the trained stimulus frequencies and enhances behavioral performance in the discrimination task (perceptual learning) (Recanzone et al., 1992). These modified cortical receptive fields are believed to underlie the enhanced behavioral discrimination that occurs after perceptual learning (Gilbert et al., 2001).

In the olfactory system, experience has also been shown to modify neural response patterns to learned odors. Associative conditioning in young rats modifies both olfactory bulb glomerular activity and mitral/tufted cell odor-evoked responses to the learned odor (Leon, 1987; Wilson and Sullivan, 1994). In mature animals, associative olfactory learning has been shown to modify odor-evoked activity in the main olfactory bulb (Viana DiPrisco and Freeman, 1985; Kendrick et al., 1992), anterior olfactory nucleus (Hamrick et al., 1993), and piriform cortex (Litaudon et al., 1997; Datiche et al., 2001). Furthermore, simple odor habituation has also been shown to modify receptive fields of both

\footnotetext{
Received Oct. 12, 2001; revised Nov. 2, 2001; accepted Nov. 6, 2001.

This work was supported by National Institute on Deafness and Other Communication Disorders Grant DC03906 and National Science Foundation Grant IBN9808149 to D.A.W. This work was performed in partial fulfillment of the M.S. thesis requirement of M.L.F.

Correspondence should be addressed to Max L. Fletcher, Department of Zoology, University of Oklahoma, Norman, OK 73019. E-mail: mick23@ou.edu.

Copyright (C) 2002 Society for Neuroscience $0270-6474 / 02 / 220001-05 \$ 15.00 / 0$
}

mitral/tufted cells and piriform cortex neurons (McCollum et al., 1991; Wilson, 2000).

Despite this wealth of data showing experience-dependent effects on olfactory system physiology, it is not known whether these learned physiological changes are translated into changes in behavioral discriminability of odorants (i.e., olfactory acuity). That is, do learning-induced changes in odor representation in the olfactory system affect the ability to discriminate those odors at a behavioral level? This is an important issue as work progresses toward quantifying spatial representations of odorant quality, usually relying on stimulation with novel odorants (Johnson et al., 1999; Rubin and Katz, 1999; Uchida et al., 2000), and correlating those spatial representations with behavioral performance in learned discrimination tasks (Linster and Hasselmo, 1999).

One difficulty in addressing the issue of whether, or how, learning changes behavioral olfactory acuity is obtaining a behavioral paradigm that allows determination of olfactory acuity to novel (or nearly novel) odors (i.e., a task that does not specifically require extensive training of the animal to the odorants before

This article is published in The Journal of Neuroscience, Rapid Communications Section, which publishes brief, peerreviewed papers online, not in print. Rapid Communications are posted online approximately one month earlier than they would appear if printed. They are listed in the Table of Contents of the next open issue of JNeurosci. Cite this article as: JNeurosci, 2002, 22:RC201 (1-5). The publication date is the date of posting online at www.jneurosci.org.

http://www.jneurosci.org/cgi/content/full/6013 
discrimination can be assessed). Toward that end, we have recently developed a habituation/cross-habituation paradigm that allows determination of olfactory acuity after minimal exposure to the odorants (Fletcher and Wilson, 2001). Odor-evoked heartrate bradycardia orienting responses are used as the behavioral measure. These responses require no initial procedural training, are expressed in response to the initial presentation of an odorant stimulus, and show relatively rapid habituation with minimal cross-habituation to molecularly dissimilar odorants (Fletcher and Wilson, 2001).

Using a homologous series of ethyl esters, the present report examined behavioral cross-habituation/generalization between odorants before and after associative conditioning. The results suggest that olfactory conditioning significantly enhances olfactory acuity for similar odorants. Given the well-described role of acetylcholine (ACh) muscarinic receptors in olfactory memory and odorant processing (Ravel et al., 1994; DeRosa and Hasselmo, 2000; Wilson, 2001b), we also examined whether these conditioning effects could be prevented by muscarinic receptor blockade.

\section{MATERIALS AND METHODS}

Subjects. Young male and female Long-Evans hooded rats (17-21 d of age) were used as subjects. Animals were born to females from Charles River Laboratories (Wilmington, MA) and were housed in polypropylene cages on a $12 \mathrm{hr}$ light/dark cycle, with food and water available ad libitum. Animal care protocols were approved by the University of Oklahoma Institutional Animal Care and Use Committee in accordance with National Institutes of Health guidelines.

Cardiac orienting response. Bilateral, subcutaneous silver chloride recording electrodes were implanted under isoflurane anesthesia. Topical lidocaine was applied at the site of electrode implantation to minimize discomfort to the animal. When animals had completely recovered from the anesthesia, they were placed in the Plexiglas testing chamber on top of a stainless-steel grid $(6 \times 15 \times 12 \mathrm{~cm})$ and allowed a $15 \mathrm{~min}$ habituation period before testing began.

A constant airstream entered the chamber from the side at 4 liters per minute, to which odorants could be added with a flow-dilution olfactometer. A vacuum line vented the odor from the chamber. Instantaneous heart rate was calculated from the interbeat interval of electrocardiogram recordings as described previously (Fletcher and Wilson, 2001) using Spike2 software (CED, Inc., Cambridge, UK). Baseline heart rate was determined from 10 consecutive heartbeats before odor presentation. Bradycardia orienting response magnitude was determined as the maximal heart rate decrease (averaged over 10 consecutive heartbeats) from baseline within $10 \mathrm{sec}$ of odor onset (Fletcher and Wilson, 2001).

Stimulus delivery. The stimulus-delivery apparatus was identical to that described by Fletcher and Wilson (2001), with humidified, filtered air passing through syringe filters saturated with specific odorants via a flow dilution olfactometer (1:10 dilution). The odorants used were ethyl butyrate (E4), ethyl valerate (E5), ethyl heptanoate (E7), ethyl octanoate (E8), isoamyl acetate (AA) (Sigma-Aldrich, St. Louis, MO), and peppermint extract.

Cross-habituation in naive animals. Animals were presented with a series of different odors to determine baseline responses. As reported previously (Fletcher and Wilson, 2001), not all animals displayed orienting responses to all odors; thus, this initial screening allowed determination of which odors to use as well as determination of initial response magnitudes. One effective odor was then pseudorandomly chosen to be the habituation stimulus. Stimuli (4 sec duration) were presented at a 30 sec interstimulus interval for 10 or 15 repetitions or until the average of the last three trial blocks was $<50 \%$ of the baseline response for that odor, depending on the experiment. After habituation, animals were presented with a molecularly dissimilar odor or a homologous odor differing by 1 or 4 carbons from the habituation odor to test for crosshabituation. Each animal was tested on a single pair of odorants. The final self- and cross-habituation magnitudes were calculated as a percentage of the initial responses.

Associative conditioning effects on cross-habituation. Conditioning took place in the chamber described above, through which footshocks were delivered. All trained animals were given 15 trials of the $\mathrm{S}^{+}$odor $(4 \mathrm{sec}$

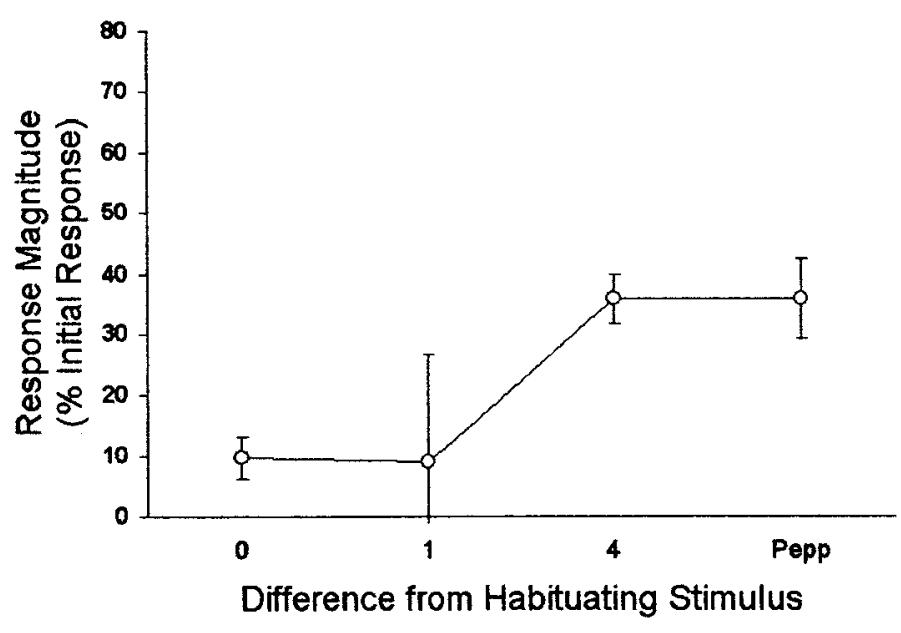

Figure 1. Discrimination of novel ethyl esters of different carbon chain lengths. The amount of self-habituation ( 0 carbon difference) was determined by the average of the last three self-trials during habituation as a percentage of the initial response. The response magnitude to the crosshabituated odor is expressed as a percentage of the initial response to the cross-habituated odor [1, 4, or peppermint (Pepp)]. Animals displayed significantly less cross-habituation to esters differing by 4 carbons or to peppermint than to esters differing by 1 carbon. Error bars denote the SE.

duration) paired with footshocks (1 sec duration, $0.5 \mathrm{~mA}$ ) at odor offset and 15 randomly interspersed trials of peppermint odor not paired with footshock $\left(\mathrm{S}^{-}\right)$. Cardiac orienting responses were not recorded during training. Immediately after conditioning, animals were returned to their home cages until testing the next day.

Like the naive animals, the animals tested the following day were given presentations of two odors to determine baseline response magnitudes. Selected odors and their relationship to the conditioned odors varied, as described below in Results. In the majority of cases, the $\mathrm{S}^{+}$odor was not selected to be the habituation odor, although no difference in selfhabituation was observed regardless of which odor was chosen. Animals were considered habituated when the average of the last three trial blocks was $<50 \%$ of the baseline response for that odor. After habituation, animals were presented with a different odor to test for cross-habituation.

Pharmacological manipulations. Before associative conditioning as described above, animals were given intraperitoneal injections of scopolamine-hydrobromide ( $\mathrm{HBr}$ ) (Sigma) or saline as a control. Fifteen minutes before conditioning began, $0.2 \mathrm{mg} / \mathrm{kg}$ scopolamine- $\mathrm{HBr}, 0.5 \mathrm{mg} / \mathrm{kg}$ scopolamine-HBr, or saline was administered. Animals were then associatively conditioned as described above and returned to their home cage until habituation/cross-habituation testing $24 \mathrm{hr}$ later. No drugs were administered during the determination of baseline response magnitudes or during the habituation phase of the experiment.

Analyses. Orienting response magnitudes and baseline heart rate were analyzed with ANOVAs and post hoc comparisons. One animal from the novel discrimination experiment and two animals from the scopolamine experiment were excluded from final analyses as statistical outliers because their final responses were $>3$ SDs from the mean of their respective groups (Barnett and Lewis, 1994).

\section{RESULTS}

Examination of cross-habituation between relatively novel similar and dissimilar odorants revealed that animals did not discriminate between ethyl esters differing by a single carbon $(n=12$ animals) but did discriminate between ethyl esters differing by 4 carbons ( $n=17)$, as evidenced by less cross-habituation, and between ethyl esters and peppermint $(n=17)$. All animals in this experiment received $104 \mathrm{sec}$ presentations of the habituating stimulus. Self-habituation (0-carbon difference from the habituating stimulus) did not vary between groups; thus these values were combined across groups for statistical analysis. As shown in Figure 1, cross-habituation levels to esters that were 1 carbon 


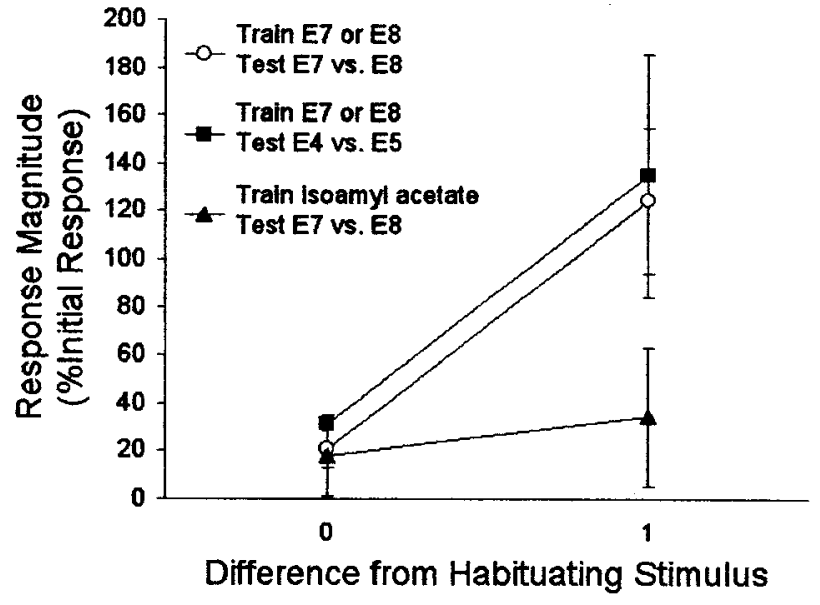

Figure 2. Discrimination of ethyl esters differing by 1 carbon after associative conditioning. Response magnitudes to the self-habituated (0) and cross-habituated (1) odors are expressed as a percentage of the initial response. One group of animals was trained to E7 or E8 and then tested for discrimination of E7 versus E8 ( $\bigcirc)$. Another group was trained to E7 or E8 and then tested with E4 versus E5 ( $\mathbf{\square})$. The last group was trained to a structurally different odor, isoamyl acetate, and then tested with E7 versus E8 ( $\mathbf{\Delta})$. After training to a similar ester, animals were capable of discriminating ethyl esters. Animals were unable to discriminate E7 versus E8 after conditioning to isoamyl acetate.

different from the habituation stimulus were similar to that of the habituation stimulus itself, whereas cross-habituation to esters that were 4 carbons different or to peppermint was substantially less (ANOVA: main effect of odor, $F_{(1,43)}=4.18, p<0.05$; post hoc Fisher's tests revealed significant differences between the response magnitude to the 4-carbon difference and peppermint stimuli from both the self-habituation/0-carbon difference and the 1-carbon difference, $p<0.05)$.

However, aversive conditioning to an ester $24 \mathrm{hr}$ before the cross-habituation test significantly enhanced discrimination between esters that were 1 carbon apart (Fig. 2). Animals were associatively conditioned with E7, E8, or AA as the $\mathrm{S}^{+}$and then tested $24 \mathrm{hr}$ later on their ability to discriminate a 1-carbon difference in esters. After $24 \mathrm{hr}$, animals received 15 habituation trials and were tested for cross-habituation. Animals conditioned with E7 or E8 as the $\mathrm{S}^{+}$were tested for discrimination of E7 versus E8 $(n=6)$ or E4 versus E5 $(n=6)$. Animals conditioned to AA as the $\mathrm{S}^{+}$were also tested for discrimination of E7 versus E8 $(n=6)$. Animals were able to discriminate ethyl esters after training to a similar ester but were unable to discriminate E7 versus E8 (1-carbon difference) after conditioning to isoamyl acetate, similar to the untrained animals described above (ANOVA: $F_{(2,30)}=10.62, p<0.05$; post hoc Fisher's tests revealed a significant difference between self-habituation and cross-habituation in both the ester-trained and ester-tested groups, $p<0.05$, but not between the amyl acetate-trained and ester-tested group).

Finally, the enhanced odorant discrimination and decreased cross-habituation produced by associative conditioning could be blocked by systemic injection of scopolamine- $\mathrm{HBr}(0 \mathrm{mg} / \mathrm{kg}, n=9$; $0.2 \mathrm{mg} / \mathrm{kg}, n=5 ; 0.5 \mathrm{mg} / \mathrm{kg}, n=6$ ) before the associative conditioning. (No drug was present during the habituation testing on the following day.) Analysis of the discrimination data revealed both a significant main effect of dose (ANOVA: $F_{(2,34)}=$ $7.09, p<0.05$ ) and a significant main effect of odor (ANOVA: $\left.F_{(2,34)}=6.39, p<0.05\right)$ on cross-habituation, with post hoc

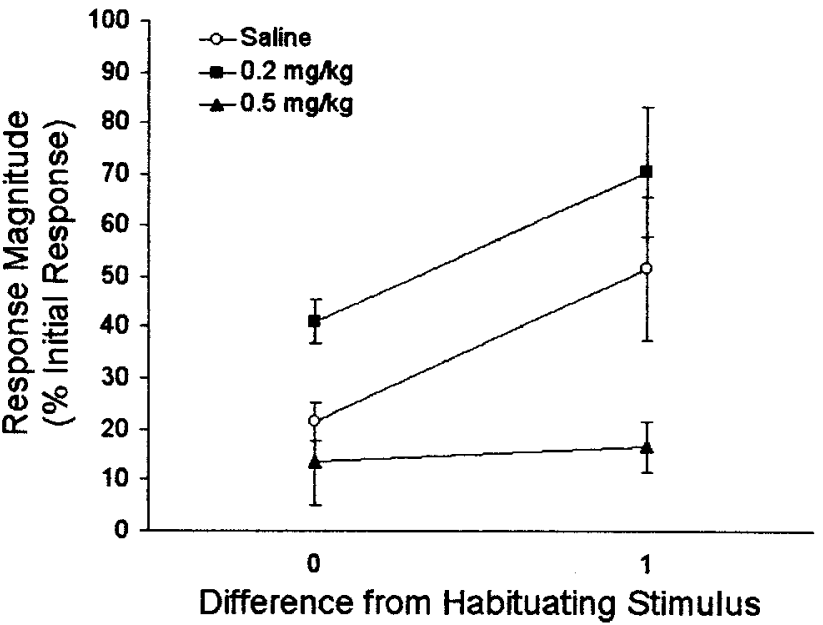

Figure 3. Discrimination of E7 and E8 after systemic injection of scopolamine- $\mathrm{HBr}$ before conditioning. Animals receiving $0.5 \mathrm{mg} / \mathrm{kg}$ scopolamine displayed significantly less discrimination and more crosshabituation of the esters than did the saline controls, while having similar levels of self-habituation. Scopolamine was only present during associative training, and not during the cross-habituation testing.

Fisher's tests confirming significantly better discrimination and less cross-habituation in the saline and $0.2 \mathrm{mg} / \mathrm{kg}$ scopolamine groups than the $0.5 \mathrm{mg} / \mathrm{kg}$ scopolamine group $(p<0.05)$. As described in Materials and Methods, in this experiment the number of habituation trials given was determined by on-line visual evaluation of the extent of habituation of odor-evoked bradycardia. Although there were no differences in the average number of habituation trials across dosages (mean habituation trials: saline, $26.2 \pm 1.2 ; 0.2 \mathrm{mg} / \mathrm{kg}$ scopolamine, $23.4 \pm 2.5 ; 0.5$ $\mathrm{mg} / \mathrm{kg}$ scopolamine, $29.7 \pm 1.4$; ANOVA: $F_{(2,18)}=1.97, p=0.17$, $\mathrm{NS}$ ), clearly the $0.2 \mathrm{mg} / \mathrm{kg}$ scopolamine animals did not attain the levels of self-habituation expressed by the saline and $0.5 \mathrm{mg} / \mathrm{kg}$ scopolamine animals, and showed minimal cross-habituation (Fig. 3). However, the $0.5 \mathrm{mg} / \mathrm{kg}$ scopolamine animals did show levels of self-habituation that were similar to those seen for the saline animals, yet these two groups displayed significantly different levels of cross-habituation.

To determine whether scopolamine affected conditioned fear responses, baseline (pre-odor) heart rates of conditioned animals with and without scopolamine were compared. Habituation testing was performed in the same context as the aversive conditioning. Animals that received conditioning had a significantly greater baseline heart rate before the discrimination test than nonconditioned animals, and there were no differences in heart rate between animals given saline or animals given scopolamine [baseline heart rate: naive, $444.74 \pm 5.70$ beats per minute (BPM); conditioned, no injection, $530.85 \pm 5.04$ BPM; conditioned-saline, $529.63 \pm 10.64 \mathrm{BPM}$; conditioned-scopolamine $(0.2 \mathrm{mg} / \mathrm{kg})$, $543.68 \pm 9.15 \mathrm{BPM}$; conditioned scopolamine $(0.5 \mathrm{mg} / \mathrm{kg})$, $530.87 \pm 8.36$ BPM; ANOVA: $\left.F_{(4,91)}=41.75, p<0.05\right]$. Post hoc Fisher's tests revealed that all conditioned groups had significantly higher baseline heart rates than the naive animals $(p<$ $0.05)$.

\section{DISCUSSION}

The present results demonstrate that olfactory acuity for similar odorants can be enhanced by previous experience with those odorants, and that acquisition of this learned enhancement can be 
impaired by the ACh muscarinic receptor antagonist scopolamine. These findings are similar to perceptual learning phenomena described in thalamocortical sensory systems (Gilbert et al., 2001).

\section{Perceptual learning}

Perceptual learning is a form of implicit memory wherein the ability to discriminate differences between stimuli (perceptual acuity) can be enhanced with training (Gibson, 1953; Gilbert et al., 2001). This change in acuity is different from an experiencedependent change in detection threshold (Wysocki et al., 1989; Dalton and Wysocki, 1996), although changes in detection threshold may also occur. Perceptual learning, as described in other sensory systems, has several characteristics (for review, see Gilbert et al., 2001): (1) the learned changes in perceptual acuity are primarily specific for the trained stimuli, although there can be some transfer to similar stimuli; (2) the learned changes are long-lasting; (3) the learned changes generally do not occur after passive stimulation, but require attention by the animal; (4) the attentional component of the learning can be mimicked by activation of cholinergic systems; and (5) the learned behavioral changes are often correlated with changes in cortical receptive fields.

The results described here match remarkably well the characteristics of perceptual learning described for other sensory systems. First, aversive conditioning to an ethyl ester enhanced discrimination of that ester from similar esters. There was some transfer of the training effect to other esters [e.g., training to ethyl octanoate (E8) enhanced discrimination of E4 from E5, but training to a completely different odorant (isoamyl acetate) did not transfer to ethyl ester discrimination]. Thus, there is some specificity in the learning effect. The lack of training specificity within esters is potentially related to the hierarchical processing of odorant features observed in optical imaging data of odorevoked spatial patterns of activity (Uchida et al., 2000). Uchida et al. (2000) suggest that odorant features may include both primary features (such as functional groups) and secondary features (such as carbon chain length). If this is correct, then perhaps associative conditioning to esters enhances coding of all esters or odors including that functional group, whereas training to a different odorant with different functional groups has no effect on esters (as shown in the current data). Current work is underway to more fully examine the nature of potential hierarchical coding in olfaction using more diverse odorant sets and odorant mixtures.

A second similarity of the current data with perceptual learning in other sensory systems is that the learning effect was longlasting, with olfactory acuity enhanced for at least $24 \mathrm{hr}$ after training. Thus, it seems unlikely that simple, rapid changes in olfactory receptor neuron sensitivity (Zufall and Leinders-Zufall, 2000) account for the findings; rather, the data suggest a change in central olfactory circuits.

Third, although the present results do not definitively identify whether associative conditioning is necessary for enhanced olfactory acuity or whether passive odor exposure is sufficient, they do demonstrate that administration of scopolamine during training, at a dose that does not impair fear conditioning to the context (Anagnostaras et al., 1999; this study), prevents experienceinduced acuity enhancement. ACh has been reported to modulate behavioral odor habituation (Hunter and Murray, 1989), performance in delayed-match-to-sample olfactory tasks (Roman et al., 1993; Ravel et al., 1994), and interference or generalization between behavioral and piriform single-unit odor representations
(DeRosa and Hasselmo, 2000; Linster et al., 2001; Wilson, 2001b); in addition, ACh reportedly modifies synaptic efficacy and plasticity in piriform cortical circuits (Hasselmo and Bower, 1992; Saar et al., 2001). Thus, the present results extend the role of ACh in olfactory memory to implicit, perceptual learning. In fact, perceptual learning may be a critical component of some of these previously described cholinergic-dependent behavioral phenomena.

Several other lines of recent evidence also suggest a role for perceptual learning in odor-discrimination performance (Rabin, 1988). For example, Cleland et al. (2002) have demonstrated recently that the ability of animals to discriminate odors is task dependent. Rats generalize between similar odorants more in simple habituation tasks (similar to what was observed here) than in rewarded tasks. The data presented here extend these findings by demonstrating that previous experience in a rewarded task (aversive conditioning) modifies a subsequent discrimination performance in an unrewarded (habituation) task. Thus, the representation of the odorant and perceptual acuity may be modified by perceptual learning and can later be expressed in other tasks and contexts.

\section{Locus of olfactory perceptual learning}

In the mammalian olfactory system, molecular/odorant features are extracted by a large family of olfactory receptors within the olfactory epithelium (Buck and Axel, 1991) and then spatially represented by odorant-specific spatial patterns of glomerular activity across the olfactory bulb (Johnson et al., 1999; Rubin and Katz, 1999; Uchida et al., 2000). Thus, for example, focal clusters of olfactory bulb glomeruli may be responsive to specific molecular functional groups and/or specific hydrocarbon chain lengths. When comparing spatial patterns evoked by different odorants, it is apparent that some odorants have highly overlapping patterns, whereas others are quite distinct. This has led to predictions about the behavioral discriminability of odorants (olfactory acuity) based on a similarity of spatial patterning (Linster and Hasselmo, 1999; Laska and Hubener, 2001). The output neurons of the olfactory bulb, mitral/tufted cells, project directly to the piriform (olfactory) cortex. Neurons within the piriform cortex have enhanced odor-discrimination abilities compared with mitral/tufted cells (Wilson, 2000, 2001a), and thus presumably also contribute significantly to behavioral olfactory acuity.

In thalamocortical sensory systems, perceptual learning is believed to be primarily a cortical phenomenon, occurring through changes of receptive fields of neurons from primary to higher order cortices (Gilbert et al., 2001). In olfaction, however, odorant responses in both the olfactory bulb and the piriform cortex can be modified by experience (Viana DiPrisco and Freeman, 1985; Leon, 1987; Kendrick et al., 1992; Wilson and Sullivan, 1994; Litaudon et al., 1997; Faber et al., 2000; Datiche et al., 2001), and plasticity in both structures is modulated by $\mathrm{ACh}$ (Elaagouby and Gervais, 1992; Ravel et al., 1994; Hasselmo and Barkai, 1995; Saar et al., 2001; Wilson, 2001b). Associative learning can modify glomerular representation of odorant features in both rats (Wilson and Sullivan, 1994) and invertebrates (Faber et al., 2000) and can also modify mitral/tufted cell odorant response patterns in an odorant-selective manner (Wilson and Sullivan, 1994). Furthermore, odorant receptive fields of piriform cortex neurons are very dynamic, expressing highly odorant-specific experience-induced changes (Wilson, 1998, 2000) in a cholinergic-dependent manner (Wilson, 2001b). Either or both of these structures may play a critical role in perceptual learning of olfactory acuity. 


\section{REFERENCES}

Anagnostaras SG, Maren S, Sage JR, Goodrich S, Fanselow MS (1999) Scopolamine and Pavlovian fear conditioning in rats: dose-effect analysis. Neuropsychopharmacology 21:731-744.

Barnett V, Lewis T (1994) Outliers in statistical data. New York: Wiley.

Buck L, Axel R (1991) A novel multigene family may encode odorant receptors: a molecular basis for odor recognition. Cell 65:175-187.

Cleland TA, Morse A, Yue EL, Linster C (2002) Behavioral models of odor similarity. Behav Neurosci, in press.

Dalton P, Wysocki CJ (1996) The nature and duration of adaptation following long-term odor exposure. Percept Psychophys 58:781-792.

Datiche F, Roullet F, Cattarelli M (2001) Expression of Fos in the piriform cortex after acquisition of olfactory learning: an immunohistochemical study in the rat. Brain Res Bull 55:95-99.

DeRosa E, Hasselmo ME (2000) Muscarinic cholinergic neuromodulation reduces proactive interference between stored odor memories during associative learning in rats. Behav Neurosci 114:32-41.

Elaagouby A, Gervais R (1992) ACh-induced long-lasting enhancement in excitability of the olfactory bulb. NeuroReport 3:10-12.

Faber T, Joerges J, Menzel R (2000) Associative learning modifies neural representations of odors in the insect brain. Nat Neurosci 2:74-78.

Fletcher M, Wilson DA (2001) Ontogeny of odor discrimination: a method to assess novel odor discrimination in neonatal rats. Physiol Behav 74:589-593.

Gibson EJ (1953) Improvement in perceptual judgments as a function of controlled practice or training. Psychol Bull 50:401-431.

Gilbert CD, Sigman M, Crist RE (2001) The neural basis of perceptual learning. Neuron 31:681-697.

Hamrick WD, Wilson DA, Sullivan RM (1993) Neural correlates of memory for odor detection conditioning in adult rats. Neurosci Lett 163:36-40.

Hasselmo ME, Barkai E (1995) Cholinergic modulation of activitydependent synaptic plasticity in the piriform cortex and associative memory function in a network biophysical simulation. J Neurosci 15:6592-6604

Hasselmo ME, Bower JM (1992) Cholinergic suppression specific to intrinsic not afferent fiber synapses in rat piriform (olfactory) cortex. J Neurophysiol 67:1222-1229.

Hunter AJ, Murray TK (1989) Cholinergic mechanisms in a simple test of olfactory learning in the rat. Psychopharmacology 99:270-275.

Johnson BA, Woo CW, Hingco EE, Pham KL, Leon M (1999) Multidimensional chemotopic responses to n-aliphatic acid odorants in the rat olfactory bulb. J Comp Neurol 409:529-548.

Kendrick KM, Levy F, Keverne EB (1992) Changes in the sensory processing of olfactory signals induced by birth in sheep. Science 256:833-836.

Laska M, Hubener F (2001) Olfactory discrimination ability for homologous series of aliphatic ketones and acetic esters. Behav Brain Res 119:193-201.

Leon M (1987) Plasticity of olfactory output circuits related to early olfactory learning. Trends Neurosci 10:434-438.

Linster C, Hasselmo ME (1999) Behavioral responses to aliphatic alde- hydes can be predicted from known electrophysiological responses of mitral cells in the olfactory bulb. Physiol Behav 66:497-502.

Linster C, Garcia PA, Hasselmo ME, Baxter MG (2001) Selective loss of cholinergic neurons projecting to the olfactory system increases perceptual generalization between similar, but not dissimilar, odorants. Behav Neurosci 115:826-833.

Litaudon P, Mouly AM, Sullivan RM, Gervais R, Cattarelli M (1997) Learning-induced changes in rat piriform cortex activity mapped using multisite recording with voltage-sensitive dye. Eur J Neurosci 9:1593-1602.

McCollum J, Larson J, Otto T, Schottler F, Granger R, Lynch G (1991) Short-latency single-unit processing in olfactory cortex. J Cogn Neurosci 3:293-299.

Rabin MD (1988) Experience facilitates olfactory quality discrimination. Percept Psychophys 44:532-540.

Ravel N, Elaagouby A, Gervais R (1994) Scopolamine injection into the olfactory bulb impairs short-term olfactory memory in rats. Behav Neurosci 108:317-324.

Recanzone GH, Merzenich MM, Schreiner CE (1992) Changes in the distributed temporal response properties of SI cortical neurons reflect improvements in performance on a temporally based tactile discrimination task. J Neurophysiol 67:1071-1091.

Roman FS, Simonetto I, Soumireu-Mourat B (1993) Learning and memory of odor-reward association: selective impairment following horizontal diagonal band lesions. Behav Neurosci 107:72-81.

Rubin BD, Katz LC (1999) Optical imaging of odorant representations in the mammalian olfactory bulb. Neuron 23:499-511.

Saar D, Grossman Y, Barkai E (2001) Long-lasting cholinergic modulation underlies rule learning in rats. J Neurosci 21:1385-1392.

Uchida N, Takahashi YK, Tanifuji M, Mori K (2000) Odor maps in the mammalian olfactory bulb: domain organization and odorant structural features. Nat Neurosci 3:1035-1043.

Viana DiPrisco G, Freeman WJ (1985) Odor-related bulbar EEG spatial pattern analysis during appetitive conditioning in rabbits. Behav Neurosci 99:964-978.

Wilson DA (1998) Habituation of odor responses in the rat anterior piriform cortex. J Neurophysiol 79:1425-1440.

Wilson DA (2000) A comparison of odor receptive field plasticity in the rat olfactory bulb and anterior piriform cortex. J Neurophysiol 84:3036-3042.

Wilson DA (2001a) Receptive fields in the rat piriform cortex. Chem Senses 26:577-584.

Wilson DA (2001b) Scopolamine enhances generalization between odor representations in rat olfactory cortex. Learn Mem 8:279-285.

Wilson DA, Sullivan RM (1994) Neurobiology of associative learning in the neonate: early olfactory learning. Behav Neural Biol 61:1-18.

Wysocki CJ, Dorries KM, Beauchamp GK (1989) Ability to perceive androstenone can be acquired by ostensibly anosmic people. Proc Natl Acad Sci USA USA 86:7976-7978.

Zufall F, Leinders-Zufall T (2000) The cellular and molecular basis of odor-adaptation. Chem Senses 25:473-481. 